\title{
Conductance statistics near the Anderson transition
}

\author{
Peter Markoš \\ Institute of Physics, Slovak Academy of Sciences, Dúbravská cesta 9, 84228 bratislava, Slovakia \\ e-mail address: markos@savba.sk
}

\section{INTRODUCTION}

Since the pioneering work of the Anderson ${ }^{1}$ we know that disorder localizes electrons. At zero temperature any small amount of the disorder localizes all electrons in the one-dimensional systems ${ }^{2}$. In higher dimension $(d>$ 2 for systems with orthogonal symmetry) weak disorder does not destroy the metallic regime. Only when the strength of the disorder increases over a certain specific value (critical disorder), the electron becomes localized. This phenomenon - transition from the metallic to the insulating regime due to an increase of disorder - is called Anderson transition ${ }^{3}$.

Scaling theory of the Anderson transition uses the conductance $g^{4}$ as the order parameter. It is supposed ${ }^{5-7}$ that the system size dependence of the conductance is determined only by the value of the conductance itself:

$$
\frac{\partial \ln g}{\partial \ln L}=\beta(g)
$$

where $\beta(g)$ is an analytical function of $g . \quad \beta$ is positive (negative) in the metallic (localized) regimes, respectively. For dimension $d>2$ the function $\beta(g)$ changes its sign, being positive for $g \gg 1$ and negative in the limit $g \rightarrow-\infty$. There is an unstable fixed point $g_{c}$ defined as the solution of $\beta\left(g=g_{c}\right)=0$. System-size independent critical conductance $g_{c}$ represents the critical point of the Anderson transition.

Relation (1) contains no information about the microscopic structure of the model. This means that the Anderson transition is universal. The form of the $\beta$ function is determined only by the physical symmetry and dimension of the system ${ }^{8}$.

Soon after the formulation of the scaling theory of localization it became clear that the conductance $g$ is not a self-averaged quantity. Reproducible fluctuations of the conductance were found both in the metallic and in the insulating regimes ${ }^{9,10}$. The knowledge of the mean value $\langle g\rangle$ is therefore not sufficient for complete description of the transport properties. One has to deal with the conductance distribution $P(g)^{11,12}$ or, equivalently, with all cummulants of the conductance. This is easier in the metallic regime, where $P(g)$ is Gaussian and the conductance fluctuations are universal ${ }^{13-21}$ and independent on the value of the mean conductance and/or the system size. The width of the distribution depends only on the dimension, physical symmetry of the system ${ }^{13}$ and on the boundary conditions ${ }^{22,23}$. In the insulator, conductance wildly fluctuates within the ensemble of macroscopically equivalent ensembles. It is the logarithm of the conduc- tance which is distributed normally in the the limit of large system size $\mathrm{e}^{11,12,17,24}$.

At present we have no complete analytical theory able to describe the conductance statistics at the critical point. Analytical results are known only for systems of dimension $d=2+\epsilon(\epsilon \ll 1)^{12,25,26}$. Expression for conductance cummulant ${ }^{25}$ enabled to estimate the shape of the critical conductance distribution $P_{c}(g)^{26} . P_{c}(g)$ is system size independent and Gaussian in the neighbor of the mean value $\langle g\rangle \sim \epsilon^{-1}$. The distribution possesses power law tail $P_{c}(g) \sim g^{-1-2 / \epsilon}$ for $g \rightarrow \infty$, and the delta-function peak $\delta(g)$. These results can not be applied to three dimensional (3D) system $(\epsilon=1)$ where $\langle g\rangle \sim 1^{27}$. Numerical simulations are therefore crucial in $3 \mathrm{D}$ system. The first systematic numerical analysis of the conductance statistics in $3 \mathrm{D}$ was done $\mathrm{in}^{27}$ and was followed by a series of papers ${ }^{28-34}$. In 2 D systems, critical conductance distribution was numerically studied in the regime of quantum Hall effect ${ }^{35}$ and in systems with the spin-orbit interaction ${ }^{28,36,37}$.

This paper reviews our recent numerical data for the conductance distribution. We address the question of the shape, universality and the scaling of the critical conductance distribution.

In numerical calculation of the conductance we suppose that two opposite sites of the sample are connected to semi-infinite perfect leads and use the multichannel Landauer formula ${ }^{38}$ which relates the conductance $g$ (in units $\left.2 e^{2} / \hbar\right)$ to the transmission matrix $t$ :

$$
g=\operatorname{Tr} t^{\dagger} t=\sum_{i=1}^{N_{\text {open }}} \cosh ^{-2} \frac{z_{i}}{2}
$$

In (2) we introduced the variables $z_{i}, i=1,2 \ldots N_{\text {open }}$, $\left(z_{1}<z_{2}<\ldots\right)$ which parametrize the eigenvalues of the matrix $t^{\dagger} t$. In the limit $L_{z}>>L, z_{i}$ converges to $2 L_{z} / \lambda_{i}$ where $\lambda_{i}$ is the $i$ th localization length of the quasi-one dimensional (quasi-1D) system ${ }^{17,27} . N_{\text {open }}$ is the number of open channels.

Owing to relation (2), the analysis of the conductance can be reduced to the calculation of the eigenvalues of the matrix $t^{\dagger} t$. The general formula for $t$ was derived in $^{39,40}$.

According to the scaling theory, critical exponents of the Anderson transition as well as conductance statistics do not depend on the microscopic details of the model. In numerical simulations, we consider the Anderson Hamiltonian

$$
\mathcal{H}=W \sum_{n} \varepsilon_{n} c_{n}^{\dagger} c_{n}+\sum_{\left[n n^{\prime}\right]} \tau_{\left[n n^{\prime}\right]} c_{n}^{\dagger} c_{n^{\prime}}
$$



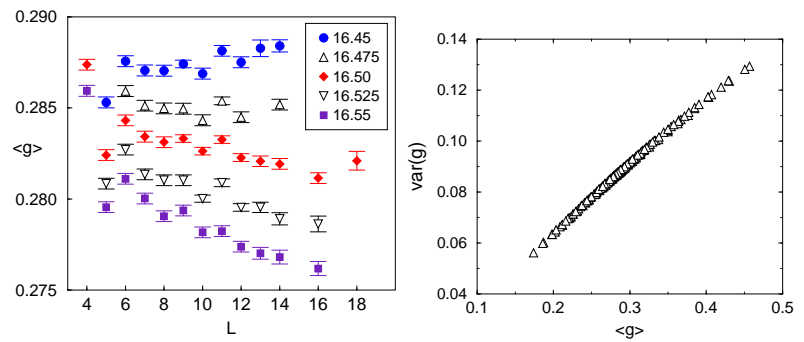

FIG. 1. Left The $L$ - dependence of the mean conductance $\langle g\rangle$ for different values of the disorder in the critical region. Data show that $16.45<W_{c}<16.55$. More exact estimation of the critical disorder was done $\mathrm{in}^{47}$. Right The unambiguous dependence var $g$ vs $\langle g\rangle$ in the critical region of the metal-insulator transition. This agrees with the single parameter scaling theory.

In (3) $n$ counts the sites of the $d$-dimensional lattice and $\left[n n^{\prime}\right]$ means nearest neighbor sites. The hopping term $\tau$ equals to unity for orthogonal systems, unless the anizotropy is considered. When spin orbit scattering is considered, $\tau$ is a $2 \times 2$ matrix $^{41,42}$. Parameter $W$ measures the strength of the disorder. For box distribution of random energies $\varepsilon_{n}(P(\varepsilon)=1$ for $|\varepsilon| \leq 1 / 2$ and $P(\varepsilon)=0$ otherwise), the 3D Anderson model (3) exhibits Anderson transition at $W_{c}=16.5$.

\section{FINITE SIZE SCALING}

As it was discussed in the Section I, the conductance $g$ is not the self-averaged quantity. To avoid statistical fluctuations, quasi-1D systems were introduced ${ }^{43}$ and the localization length $\gamma$ is calculated instead of the conductance. In the limit $L_{z} / L \rightarrow \infty\left(L_{z}\right.$ and $L$ are the length and the width of the system, respectively) $\gamma$ is a self-averaged quantity which converges to its mean value. Finite size scaling is then used $^{44}$ for the analysis of the disorder and the system width dependence of $\gamma$. It is assumed that the variable $\Lambda=\gamma / L$ is a function of only one parameter: $\Lambda(L, W)=\Lambda(L / \xi(W))$. Here $\xi=\xi(W)$ is the correlation length which diverges in the vicinity of the critical point $\xi(W)=\left|W-W_{c}\right|^{-\nu}$. Critical exponents $\nu$ and $s=(d-2) \nu$ characterize the critical behavior of the localization length and of the conductance, respectively ${ }^{5}$.

Finite size scaling analysis of the quasi-1D data enabled to test the universality of the Anderson transition ${ }^{45}$ and provided us with the more accurate estimation of the critical exponent $\nu \approx 1.57^{46}$.

\section{A. Scaling of the mean conductance}

Verification of the scaling theory of the localization requires the proof of the universal scaling of the mean conductance and of the entire conductance distribution in the critical regime. Single parameter scaling of various mean values, $\langle g\rangle$, $\exp \langle\ln g\rangle$, and $1 /\left\langle g^{-1}\right\rangle$, was proved numerically for the 3D Anderson model ${ }^{47}$. Statistical ensembles of more than $N_{\text {stat }} \geq 10^{6}$ cubes of the size from $4^{3}$ to $18^{3}$ were collected for various values of the disorder $W$. Fig. 1 shows typical data for the system size dependence of the mean conductance. In agreement with (1), $\langle g\rangle$ increases (decreases) with the system size in the metallic (localized) regime. By the use of the general fitting procedure ${ }^{46}$, the critical disorder and the critical exponent $\nu \approx 1.57$ were obtained.

Data in fig. 1 confirm that the variance, var $g=$ $\left\langle g^{2}\right\rangle-\langle g\rangle^{2}$ is an unambiguous function of the mean $\langle g\rangle$ in the critical regime. This supports, but still does not prove the single parameter scaling theory. General proof of the theory requires verification of the single parameter scaling of all conductance cummulants. This is numerically impossible since higher cummulants are fully determined by rare events with very large values of the conductance.

\section{B. Scaling of the conductance distribution}

As higher cummulants are not treatable numerically, we test the scaling of the conductance distribution by the analysis of the scaling of percentiles $g_{\alpha}{ }^{34}$. Percentile $g_{\alpha}$ is defined as

$$
\alpha=\int_{0}^{g_{\alpha}} P(g) \mathrm{d} g .
$$

Owing to (4), the probability to find $g<g_{\alpha}$ equals to $\alpha$. Of course, the percentile $g_{\alpha}$ is a function of disorder and system size: $g_{\alpha}=g_{\alpha}(L, W)$. Single parameter scaling of percentiles has been proved for several values of $\alpha^{34}$.
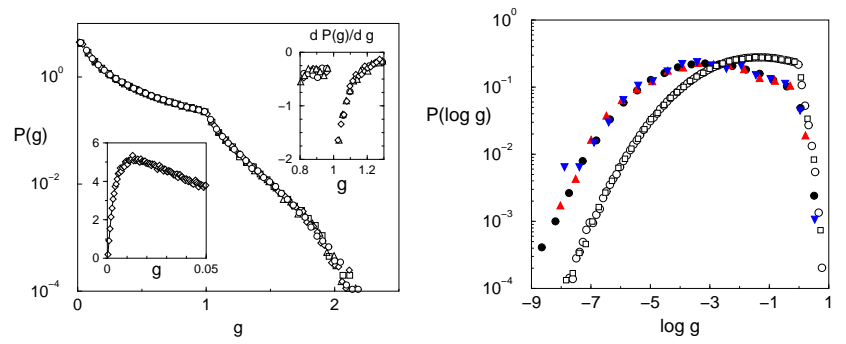

FIG. 2. Left Critical conductance distribution $P_{c}(g)$ of the 3D Anderson model, obtained for statistical ensembles of $N_{\text {stat }}=10^{6}$ samples of the size $10^{3}-18^{3} . P_{c}(g)$ is system size independent and decreases faster than exponentially when $g>1$. The main properties of the critical distribution are shown in insets: Left inset shows in details the small - $g$ behavior and proves that the distribution decreases to zero as $g \rightarrow 0^{+}$. Right inset shows the discontinuity of the derivation $\mathrm{d} P(g) / \mathrm{d} g$ at $g=1$. Right figure presents the distribution $P_{c}(\ln g)$ at the critical point for the three dimensional $\left(W_{c}=16.5\right.$, open symbols $)$ and four dimensional (4D) $\left(W_{c}=34.3\right.$, full symbols $)$ systems of various system size. 

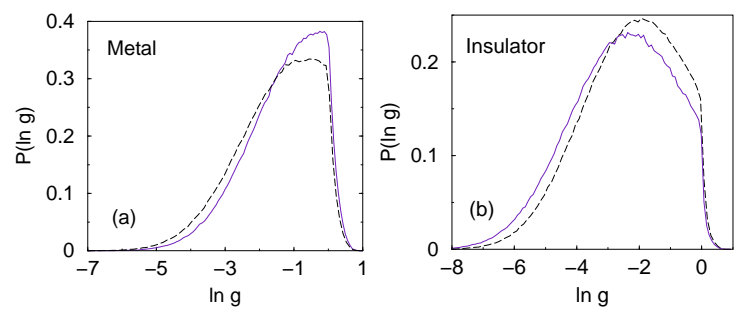

FIG. 3. Conductance distribution for ensembles of cubes $6^{3}$ (dashed lines) and $12^{3}$ (solid line): (a) The metallic regime $W=15.4$ (the critical disorder is $W_{c}=16.5$ ). Distribution $P(g)$ moves toward higher values of the conductance as the system size increases and $P(g)$ becomes Gaussian when $L \rightarrow \infty$. (b) Insulator $W=17.6$ Mean conductance decreases as the system size increases and the distribution of the $\ln g$ becomes Gaussian in the limit $L \rightarrow \infty$.

Suppose that $g_{\alpha}$ and $g_{\beta}(\alpha<\beta)$ obey the single parameter scaling. Then $g_{\gamma}(\alpha<\gamma<\beta)$ scales, too. Therefore, in contrast to the analysis of the conductance cummulants, it is enough to analyze only a few percentiles. Next, if $g_{\alpha}$ and $g_{\beta}$ scale, then the difference $g_{\beta}-g_{\alpha}$ scales. Scaling of percentiles assures thus the scaling of the entire conductance distribution. Of course, this analysis is not applicable to the limit $\alpha \rightarrow 1$, because available statistical ensembles are never big enough to provide us with sufficient information about the tail of the distribution.

\section{CRITICAL CONDUCTANCE DISTRIBUTION}

At present, we have no analytical description of the critical conductance distribution in 3D systems. Analytical results were obtained only for the conductance cummulants in the dimension $d=2+\epsilon$ close to the lower critical dimension $(\epsilon \ll 1)^{25}$. In spite of the non-universality of higher order conductance cummulants

$$
\left\langle\delta g^{n}\right\rangle= \begin{cases}\epsilon^{n-2} & n<n_{0}=\epsilon^{-1} \\ \sim L^{\epsilon n^{2}-n} & n>\epsilon^{-1}\end{cases}
$$

the critical distribution $P_{c}(g)$ was shown to be universal and $L$-independent in the limit $L \rightarrow \infty^{26}$. However, theoretical analysis of the form of the critical distribution, is applicable only in the limit of very small $\epsilon^{27}$.

\section{A. The form of the critical conductance distribution}

All what we know about the $P_{c}(g)$ in $3 \mathrm{D}$ is based on the numerical data. In Fig. 2 we present $P_{c}(g)$ for the 3D Anderson model. Data confirm that the critical conductance distribution is system size independent, as required ${ }^{12}$. The shape of $P_{c}(g)$ differs considerably from the conductance distribution in the metallic and in the insulating regimes. To explain the typical properties of the critical conductance distribution, we use our knowledge about statistical properties of parameters $z(2)$.

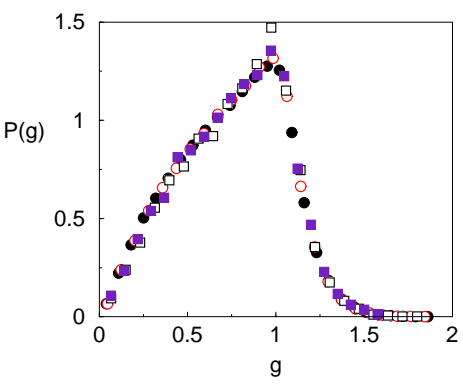

FIG. 4. Comparison of the critical conductance distribution of two different 2D models with spin-orbit coupling: full symbols Ando model ${ }^{42}$ open symbols Evangelou-Ziman model $^{41}$. Squares $40 \times 40$ and $80 \times 80$ were simulated to prove the system size independence of the critical distribution. Note also the non-analytic behavior for $g=1$.

Decrease of $P_{c}(g)$ to zero when $g \rightarrow 0$ is visible when very large statistical ensembles are studied (left inset of Fig. 2). Due to Eq. (2), small conductance means that $z_{1}$ is large. From numerical data we know that the distribution $P\left(z_{1}\right)$ is similar to Wigner surmises and decreases as $\exp -z_{1}^{2}$ for large $z_{1}$. Consequently, $\ln P_{c}(\ln g)$ decreases as $-\ln ^{2} g$ (right figure in Fig. 2), and guarantees that $\lim _{g \rightarrow 0} P_{c}(g)=0^{27,30}$.

Large $g$ behavior of $P_{c}(g)$ is determined by the chance that many parameters $z_{i}$ are small. Statistical analysis of parameters $z_{i}$ showed that for $i>1$ the distribution $P\left(z_{i}\right)$ is Gaussian with mean value $\left\langle z_{i}\right\rangle \propto \sqrt{i}$ and var $z_{i} \propto\left\langle z_{i}\right\rangle^{-130}$. The probability to find a sample with small value of higher $z_{i}$ is therefore very small: $P_{c}(g \approx i)$ decreases as $\exp -i^{3 / 230}$. Fig. 2 indeed shows very fast decrease of the probability $P_{c}(g)$ for $g>1$. The chance to have $g>1$ is only $3 \%$. Probability to find large values of $g$ drastically decreases: we found that in the ensemble of $10^{7}$ samples $(L=10)$ only 470 samples have $g>2$ and only one sample has $g>3^{33}$. The analysis of the contribution of the first two channels is therefore sufficient for the understanding of the qualitative properties of the critical distribution.

Numerical data also show that the critical distribution is non-analytical at $g=1$. Right inset of Fig. 2 shows the discontinuity of the first derivative $\mathrm{d} P_{c}(g) / \mathrm{d} g$. The same non-analyticity was found in the 4D systems (right figure 2 ), in the unitary ${ }^{29}$ and symplectic (Fig. 5) systems, and also in the weakly disordered quasi-1D systems ${ }^{48,49}$.

Present description of the critical distribution is based on the analysis of statistical properties of parameters $z$. It is applicable to any system, for which the mean values of parameters $z$ are of the order of unity. Then only a few (two, or three) channels contribute to the conductance. This analysis is, however, not applicable to systems close to the lower critical dimension $d=2+\epsilon$. Here, the mean conductance $\langle g\rangle \sim \epsilon^{-1}$, which means that number of channels which contribute to the conductance, is large, $\sim \epsilon^{-1}$. It is therefore no surprise that the critical conductance distribution found in $^{26}$ differs from that shown in fig. 2. 


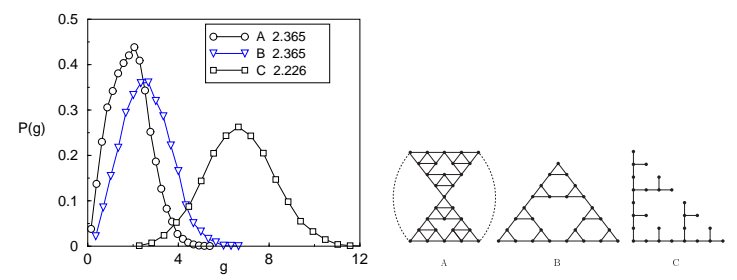

FIG. 5. Critical conductance distribution for bifractal lattices. Legend gives spectral dimension. 5th generation of fractals was used to calculate the distribution. Bifractal is linear along the $z$ axis and fractal in a plane perpendicular to $z$. Right figure shows the structure of the fractal (the 3rd generation) cross section. All fractals have the same fractal dimension $d_{\mathrm{f}}=\ln 3 / \ln 2$. Spectral dimension $d_{\mathrm{s}}=\ln 9 / \ln 5$ for fractals $A$ and $B$, and $=\ln 9 / \ln 6$ for fractal C. Note that although bifractals $\mathrm{A}$ and $\mathrm{B}$ have the same both fractal and spectral dimensions, they posses different critical conductance distribution. $P_{c}(g)$ depends on the lattice topology ${ }^{51}$

\section{B. Conductance distribution in the critical regime}

Fig. 3 shows that the typical properties of the critical conductance distribution hold also for $P(g)$ in the neighbor of the critical point. This is because the statistical properties of parameters $z$ depend continuously on the disorder in the critical regime. Critical properties of $P(g)$ survive until the system size $L$ exceeds the correlation length $\xi$. Only when $\xi \ll L$ the distribution typical for the metallic or the insulating regime can be observed.

\section{Universality}

Single parameter scaling theory of localization supposes that the critical conductance distribution is universal. Its form does not depend on the microscopic details of the model. As an example, we present in Fig. 4 the critical conductance distribution for two 2D models with spin-orbit scattering: Evangelou-Ziman model ${ }^{41}$ and Ando model ${ }^{42}$. In spite of the different microscopic definition of both models, $P_{c}(g)$ is universal ${ }^{28}$. Universality of $P_{c}(g)$ with respect to various distributions of random energies $\varepsilon_{n}$ was confirmed in $^{28}$ and $^{32}$.

As was shown already in fig. 2, the shape of $P_{c}(g)$ depends on the dimension of the model. Ref. ${ }^{29}$ confirmed that also the physical symmetry influences the form of $P_{c}(g)$. Less expectable was the observation 22,31 that $P_{c}(g)$ as well as the spectral statistics ${ }^{50}$ depend on the boundary conditions in the transversal direction. Nevertheless, this is consistent with the original definition of the conductance as a measure of the sensitivity of the energy spectrum of the system to the change of the boundary conditions ${ }^{4}$.

For completeness, let us note that the critical conductance distribution depends also on other parameters of the model: lattice topology ${ }^{51}$, anizotropy ${ }^{37}$ and, of course, on the length of the system. We believe that these non-universalities could be compensated by the change of another model parameter (see, for instance ${ }^{37}$, where the anizotropy is compensated by the length of the system).

\section{Dimension dependence}

Right figure in Fig. 2 compares the critical conductance distribution for $3 \mathrm{D}$ and $4 \mathrm{D}$ cubes. As supposed, the maximum of $P_{c}(g)$ for $4 \mathrm{D}$ is shifted toward smaller conductances, because the critical disorder increases as the spatial dimension increases and higher disorder means lower mean conductance ${ }^{5,26}$. Qualitatively, however, both distributions are very similar: $P_{c}(\ln g)$ decreases as $\exp \left[-\ln ^{2} g\right]$ for $\ln g \rightarrow-\infty$ and possesses the non-analyticity at $\ln g=0$. This similarity is not surprising, because the form of the distribution is determined mostly by the statistics of $z_{1}$ and $z_{2}$, which are qualitatively similar in $3 \mathrm{D}$ and $4 \mathrm{D}^{30}$.

Surprisingly, the relation (5) seems to hold also for $\epsilon=1$ and 2 , at least for the first two cummulants. We obtained numerically that $\langle g\rangle=0.285$ for $3 \mathrm{D}$ and 0.135 for $4 \mathrm{D}$, so that $\langle g\rangle_{3 \mathrm{D}} \approx 2\langle g\rangle_{4 \mathrm{D}}$. For the second cummulants we found var $g_{3 \mathrm{D}} \approx \operatorname{var} g_{4 \mathrm{D}} \approx 0.17^{51}$.

More interesting is the investigation of the $P_{c}(g)$ in the systems of dimension $2+\epsilon^{51}$. As we are not able to create the $d$-dimensional hyper-cubes with non-integer $d$ in computers, we simulated the transport on bifractal latices $^{52,51}$. Bifractals are linear along the propagation direction and possess the fractal lattice in the cross section (fig. 5). We proved that the critical exponent $\nu$ depends only on the spectral dimension of the lattice. Mean conductance, var $g$ and the critical distribution $P_{c}(g)$ depend, however, on the lattice topology. For instance, fig. 5 shows that bifractals A and B have the same spectral dimension, but different critical distribution. This is the reason why obtained data can not be used for the verification of relations (5).

In Fig. 5 we present $P_{c}(g)$ for three different bifractals. As expected, $\langle g\rangle$ increases and the distribution converges to Gaussian when $\epsilon \rightarrow 0$. However, we found neither the $\delta$ - function peak at $g=0$ nor the power-law tail of the distribution for $g \gg\langle g\rangle$, predicted by the theory ${ }^{26}$.

\section{CONDUCTANCE DISTRIBUTION IN NON-CRITICAL REGIME}

Although we have no analytical theory of the conductance statistics in the critical regime, we can learn some typical properties of the conductance distribution from the analysis of the quasi-1D weakly disordered systems ${ }^{48}$. Starting from the Dorokhov - Mello-PereyraKumar equation ${ }^{15}$ for the probability distribution of parameters $z$, the conductance distribution $P(g)$ can be calculated. $P(g)$ depends on the length of the system. 

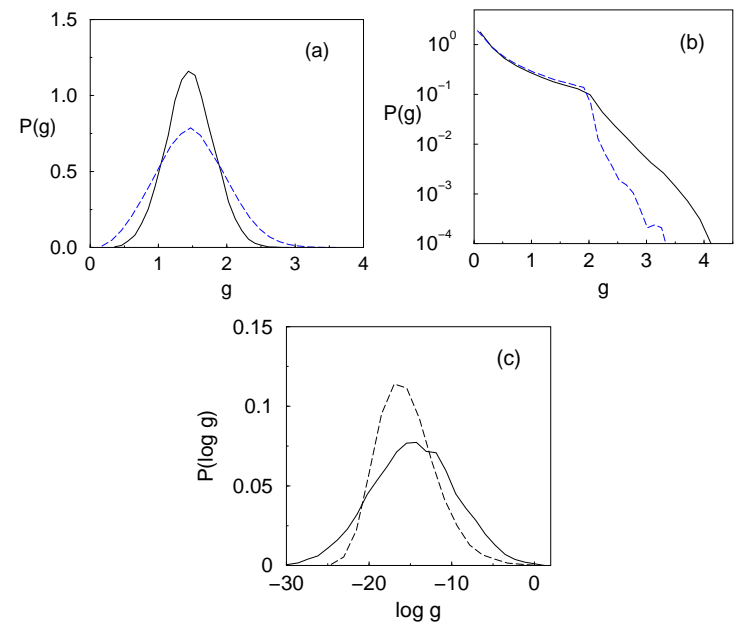

FIG. 6. Comparison of the conductance distribution of the 3D (dashed lines) and quasi-1D (solid line). (a) Metallic $(\langle g\rangle>1)$ (b) critical $(\langle g\rangle \approx 1)$ and (c) localized $(\langle g\rangle \ll 1)$ regimes are shown. In the quasi-1D systems, the strength of the disorder is fixed to $W=4$ and the length of the system is tuned to obtain the same mean conductance as in the $3 \mathrm{D}$ system.
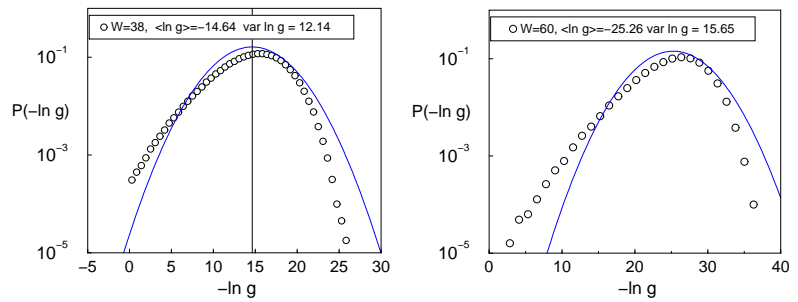

FIG. 7. Distribution of $-\ln g$ for strongly localized regime. Note that the distribution is not symmetric. It possesses the long tail for small values of the argument, and decreases much faster on the opposite side. Solid lines are a Gaussian distribution with the mean and variance found from numerical data. Size of the cubes is $L=10$. Left: $W=38$. Note that the sharp decrease of the distribution at $\ln g=0$, discussed in Sect. 3.1, is still present ${ }^{48}$. Right: $W=60$.

For short samples, $P(g)$ is Gaussian ${ }^{17}$ (see also fig. 6a). When the length of the system increases, the conductance decreases and the system passes from the metallic regime into the localized one. For the intermediate system length, where $\langle g\rangle \approx 1, P(g)$ is expected to be qualitatively similar to the critical conductance distribution ${ }^{48}$. This is shown in Fig 6, where we compare the conductance distribution of quasi-1D and 3D systems of the same mean conductance.

Fig. 6 also shows that the analogy between 3D and quasi-1D systems is not exact. To understand quantitative differences between the quasi-1D and 3D systems, we analyzed the spectrum of parameters $z$. In the metallic regime, the difference between $3 \mathrm{D}$ and quasi-1D systems is only in the value of var $g^{13} . P(g)$ is Gaussian and the spectrum of parameters $z$ is linear $\left\langle z_{i}\right\rangle \propto i^{14,17}$ indepen- dently on the dimension of the system ${ }^{54}$. In contrast to the metallic regime, the spectrum of $z$ becomes dimension dependent in the critical region. Both quasi- $1 \mathrm{D}^{53,54}$ and $3 \mathrm{D}$-dimensional ${ }^{49}$ numerical studies confirmed that at the critical point

$$
\left\langle z_{i}\right\rangle^{d-1} \propto i
$$

$(d>2)$. Owing to (6), the difference $\Delta=\left\langle z_{2}-z_{1}\right\rangle$ is smaller that $\left\langle z_{1}\right\rangle$ in $3 \mathrm{D}$, while it equals to $\left\langle z_{1}\right\rangle$ in the quasi-1D system. The contribution of the second channel is therefore more important in 3D than in the quasi-1D. This explains longer tail of the distribution in the 3D system (fig. 6b).

It is commonly believed that the distribution $P(\ln g)$ is Gaussian in the insulating phase, independently on the dimension of the system. This is,however, not true. The spectrum of $z$ depends namely on the dimension of the system also in the localized regime. For the 3D systems it was proved numerically ${ }^{49,54}$ that the difference $\Delta=\left\langle z_{2}-z_{1}\right\rangle$ is constant, independent on the disorder and on the system size. Therefore the second channel influences always the statistics of the conductance. More than its contribution to the value of the conductance it is important that constant value of $\Delta$ prevents the distribution $P\left(z_{1}\right)$ to develop into the Gaussian form. While the values $z_{1} \ll\left\langle z_{1}\right\rangle$ are still possible, the probability to find systems with much higher values $z_{1} \gg\left\langle z_{1}\right\rangle$ is strongly suppressed. The distribution $P\left(z_{1}\right)$ is therefore not symmetric. The same is true for the distribution $P(-\ln g)$ (Fig. 7) which possesses a long tail for small values of $|\ln g|$ and decreases much faster than Gaussian for $|\ln g| \rightarrow \infty$.

Note that in weakly disordered quasi-1D systems $\Delta \sim$ $\left\langle z_{1}\right\rangle$. The distance $\left\langle z_{2}-z_{1}\right\rangle$ is much larger than the width of the distribution $P\left(z_{1}\right)$. Higher channels therefore do not influence the distribution of $z_{1}$ and $P \ln g$ is Gaussian.

\section{CONCLUSION}

We reviewed recent progress in numerical studies of the statistics of the conductance in the critical regime. Numerical analysis confirms that the conductance distribution in 3D Anderson model obeys single parameter scaling. Analysis of the statistics of the eigenvalues of the transmission matrix enables us to understand the main features of the conductance distribution in the critical regime. Although we still have no analytical description of the conductance statistics in the critical regime, we hope that results of numerical experiments will inspire theoreticians to formulate the general analytical theory of the Anderson transition. 
${ }^{1}$ P. W. Anderson: Phys. Rev. 109, 1492 (1958)

${ }^{2}$ N. F. Mott, W. D. Twose: Adv. Phys. 10, 107 (1961)

${ }^{3}$ N. F. Mott, E. A. Davis: Electron processes in noncrystalline materials, Clarendon Press, Oxford 1979

${ }^{4}$ J. T. Edwards, D. J. Thouless: J. Phys. C 5, 807 (1972); D. C. Licciardello, D. J. Thouless: J. Phys. C 8, 4157 (1975)

${ }^{5}$ F. Wegner: Z. Phys. B 25, 327 (1976)

${ }^{6}$ E. Abrahams, P. W. Anderson, D. C. Licciardello, T. V. Ramakrishnan: Phys. Rev. Lett. 42, 673 (1979)

${ }^{7}$ L. P. Gorkov, A. I. Larkin, D. E. Khmelnitskii: JETP Lett. 30, 228 (1979)

${ }^{8}$ B. Kramer, A. MacKinnon: Rep. Prog. Phys 56, 1469 (1993)

${ }^{9}$ S. Washburn, R. A. Webb: Adv. Phys. 35, 375 (1986)

${ }^{10}$ A. B. Fowler, J. J. Wainer, R. A. Webb: IBM J. Res. Dev. 32, 372 (1988)

${ }^{11}$ P. W. Anderson, D. J. Thouless, E. Abrahams, D. S. Fisher: Phys. Rev. B 22, 3519 (1980)

12 B. Shapiro: Philos. Mag. B 56, 1032 (1987); Phys. Rev. Lett. 651510 (1990)

13 P. A. Lee, A. D. Stone: Phys. Rev. Lett. 55, 1622 (1985); P. A. Lee, A. D. Stone, H. Fukuyama: Phys. Rev. B 35, 1039 (1987)

${ }^{14}$ I. Imry: Europhys. Lett. 1, 249 (1986)

${ }^{15}$ O. N. Dorokhov: JETP Lett. 36, 318 (1982); P. A. Mello, P. Pereyra, N. Kumar: Ann. Phys. (NY) 181, 290 (1988)

${ }^{16}$ C. W. J. Beenakker: Rev. Mod. Phys. 69, 731 (1997)

17 J.-L. Pichard: 'Random transfer matrix theory and conductance fluctuations'. In: Quantum Coherence in Mesoscopic Systems NATO ASI Ser. B: Physics vol. 254 ed. by B. Kramer (Plenum Press, 1991)

${ }^{18}$ P. M. Bell, A. MacKinnon: J. Phys.: Condens. Matt. 6, 5423 (1994)

19 N. Giordano: Phys. Rev. B 38, 4746 (1988)

${ }^{20}$ J.-L. Pichard, N. Zannon, I. Imry, A. D. Stone: J. Phys. France 51, 587 (1990)

${ }^{21}$ Y. Avishai, J.-L. Pichard, K. A. Muttalib: J. Phys. France 3, 1387 (1991)

${ }^{22}$ D. Braun, E. Hofstetter, G. Montambaux, A. MacKinnon: Phys. Rev. B 64, 155107 (2001)

${ }^{23}$ M. Rühländer, P. Markoš, C. M. Soukoulis: Phys. Rev. B 64, $172202(2001)$

${ }^{24}$ P. Markoš, B. Kramer: Ann. Physik (Leipzig) 2, 339 (1993)

${ }^{25}$ B. L. Altshuler, V. E. Kravtsov, I. V. Lerner: Sov. Phys. JETP 64, 1352 (1986); JETP Lett. 43, 441 (1986)

${ }^{26}$ A. Cohen, B. Shapiro: Int. J. Mod. Phys. B 6, 1243 (1992)

${ }^{27}$ P. Markoš, B. Kramer: Philos. Mag. B 68, 357 (1993)

${ }^{28}$ P. Markoš: Europhys. Lett. 26, 431 (1994)

${ }^{29}$ K. Slevin, T. Ohtsuki: Phys. Rev. Lett. 78, 4083 (1997);

${ }^{30}$ P. Markoš: Phys. Rev. Lett. 83, 588 (1999)

${ }^{31}$ K. Slevin, T. Ohtsuki, T. Kawarabayashi: Phys. Rev. Lett. 84, 3915 (2000)

${ }^{32}$ K. Slevin, T. Ohtsuki: Phys. Rev. B 63, 45108 (2001)

${ }^{33}$ M. Rühländer, P. Markoš, C. M. Soukoulis: Phys. Rev. B 64, $212202(2001)$

${ }^{34}$ K. Slevin, P. Markoš, T. Ohtsuki: cond-mat/0208157

${ }^{35}$ Z. Wang, B. Jovanovic, D. H. Lee: Phys. Rev. Lett. 77, 4426 (1996); V. Plerou, Z. Wang: Phys. Rev. B 58, 1967 (1998); X. Wang, Q. Li, C. M. Soukoulis: Phys. Rev. B 58, R3576 (1998)
${ }^{36}$ P. Markoš: J. Phys. I France 4, 551 (1994)

${ }^{37}$ M. Rühländer, P. Markoš, C. M. Soukoulis: Phys. Rev. B 64, 193103 (2001)

38 R. Landauer: IBM J. Res. Dev. 1, 223 (1957); R. Landauer: Phil. Mag. 21, 683 (1970); E. N. Economou, C. M. Soukoulis: Phys. Rev. Lett. 46, 618 (1981)

39 T. Ando: Phys. Rev. B 44, 8017 (1991)

40 J. B. Pendry, A. MacKinnon, P. J. Roberts: Proc. P. Soc. London A 437, 67 (1992)

${ }^{41}$ S. N. Evangelou, T. Ziman: J. Phys. C 20, L235 (1987)

42 T. Ando: Phys. Rev. B 40, 5325 (1989)

43 J.-L. Pichard, G. Sarma: J. Phys. C 14, L127 (1981); ibid, L167

${ }^{44}$ A. MacKinnon, B. Kramer: Phys. Rev. Lett. 47, 1546 (1981)

${ }^{45}$ B. Bulka, B. Kramer, A. MacKinnon: Z. Phys. B: Condens. Matt. 60, 13 (1985); B. Kramer, K. Broderix, A. MacKinnon, M. Schreiber: Physica A 167, 163 (1990); A. MacKinnon: J. Phys.: Condens. Matt 6, 2511 (1994)

${ }^{46}$ K. Slevin, T. Ohtsuki: Phys. Rev. Lett. 82, 382 (1999)

${ }^{47}$ K. Slevin, P. Markoš, T. Ohtsuki: Phys. Rev. Lett. 86, 3594 (2001)

${ }^{48}$ K. A. Muttalib, P. Wölfle: Phys. Rev. Lett. 83, 3013 (1999); V. A. Gopar, K. A. Muttalib, P. Wölfle: condmat/0205643; K. A. Muttalib, P. Wölfle, A. García-Martín, V. A. Gopar: cond-mat/0210293

${ }^{49}$ P. Markoš: Phys. Rev. B 65, 104207 (2002)

${ }^{50}$ D. Braun, G. Montambaux, M. Pascaud: Phys. Rev. Let. 81, 1062 (1998)

${ }^{51}$ I. Travěnec, P. Markoš: Phys. Rev. B 65, 113109 (2002)

${ }^{52}$ M. Schreiber, H. Grußbach: Phys. Rev. Lett. 76, 1687 (1996)

${ }^{53}$ P. Markoš, M. Henneke: J. Phys.: Condens. Matt. 6, L765 (1994)

${ }^{54}$ P. Markoš: J. Phys.: Condens. Matt. 7, 8361 (1995) 